Revue des patrimoines

$24 \mid 2014$

Architecture et urbanisme de villégiature : un état de la recherche

\title{
« La petite mer d'Enghien » : un site pour une villégiature parisienne
}

\section{Sophie Cueille}

\section{(2) OpenEdition}

\section{Journals}

Édition électronique

URL : http://journals.openedition.org/insitu/11096

DOI : $10.4000 /$ insitu. 11096

ISSN : 1630-7305

Éditeur

Ministère de la Culture

\section{Référence électronique}

Sophie Cueille, « "La petite mer d'Enghien » : un site pour une villégiature parisienne », In Situ [En ligne], 24 | 2014, mis en ligne le 17 juillet 2014, consulté le 07 juillet 2020. URL : http://

journals.openedition.org/insitu/11096; DOI : https://doi.org/10.4000/insitu.11096

Ce document a été généré automatiquement le 7 juillet 2020.

\section{(c)}

In Situ Revues des patrimoines est mis à disposition selon les termes de la licence Creative Commons Attribution - Pas d'Utilisation Commerciale - Pas de Modification 4.0 International. 


\section{«La petite mer d'Enghien »: un site pour une villégiature parisienne}

\section{Sophie Cueille}

\section{(fig. 1)}

Figure 1

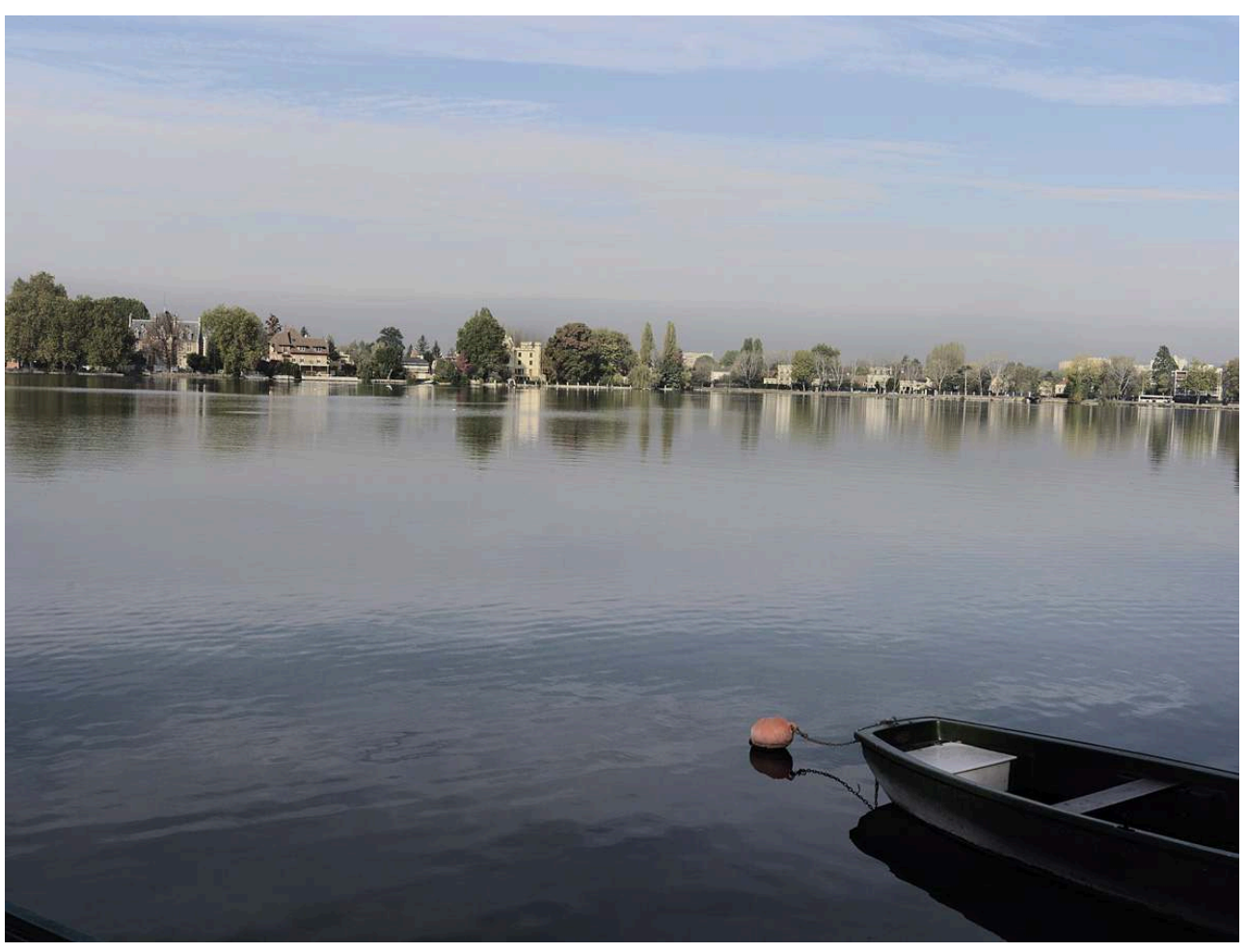

Vue actuelle du lac.

Phot. Vialles, Jean-Bernard. (c) Conseil régional d'île-de-France, service Patrimoines et Inventaire, 2008. (base Mémoire : 20089500581) 
2 Le territoire de la ville d'Enghien est situé en contrebas de Montmorency et fut le domaine seigneurial des Montmorency jusqu'en 1633, date à laquelle il entre par alliance dans le domaine des princes de Condé1. En raison du titre de duc d'Enghien détenu par les Condé dès le XVI ${ }^{e}$ siècle, affirmé à nouveau par Louis XIV en 1689, le duché de Montmorency est transformé en duché d'Enghien.

3 L'étang qui forme le creux de la vallée, réunissant les cours des différents rus, porte alors le nom d'étang d'Enghien. Hormis un moulin et un hameau au lieu-dit d'Ormesson, le site est vierge de toute habitation jusqu'à l'implantation des premiers bâtiments thermaux.

C'est sur les bords de cet étang que le jeune prêtre de l'Oratoire de Montmorency, Louis Cotte (1740-1815) découvre en 1766 la présence d'eaux de nature sulfureuse. Scientifique reconnu, il présente plusieurs mémoires à l'Académie royale des sciences et contribue ainsi à forger la renommée des eaux d'Enghien. À la toute fin du XVIII ${ }^{\mathrm{e}}$ siècle, avec Guillaume Le Veillard, sont lancées les prémices d'une véritable exploitation thermale. Ce dernier étant guillotiné en 1794, la concession est transmise en 1803 par sa veuve à Mme Gauthier qui fait construire au bord du lac le premier établissement thermal.

5 Mais l'on peut considérer que l'année 1820 est décisive, avec l'étude menée sur ordre du gouvernement pour mettre en œuvre une analyse générale des eaux du royaume. Le but en est à la fois économique et politique, la stratégie étant d'attirer les étrangers vers les eaux du pays. Enghien est alors présenté comme un lieu prometteur et qui prendra bientôt « une place distinguée parmi les eaux minérales le plus en usage en France $^{2} »$. Des guérisons fameuses, dont celle de Louis xviII venu prendre les eaux sur les conseils du médecin en chef de l'hôpital Saint-Louis, contribuent à établir la renommée médicale des eaux d'Enghien. L'étang s'élève peu à peu au rang de lac et ses berges sont raffermies. Alexandre Dumas, qui fréquenta les rives du lac, a décrit dans ses Mémoires les lieux avant leur métamorphose en station thermale élégante :

Le lac d'Enghien était à cette époque un lac tout simplement, un vrai lac, un peu boueux pour un lac, pas assez pour un étang. Il était couvert de joncs, de nénuphars, au milieu desquels jouaient les plongeons, caquetaient les poules d'eau, barbotaient les canards sauvages.

\section{La perception du lac au début du XIX $\mathrm{X}^{\mathrm{e}}$ siècle : entre nature recomposée et nature romantique}

6 Pour mieux comprendre l'histoire du site du lac d'Enghien, il convient de retracer brièvement la place du lac dans la composition des parcs de la fin du XVIII ${ }^{\mathrm{e}}$ et du début du $\mathrm{XIX}^{\mathrm{e}}$ siècle mais aussi d'évoquer le rôle qu'il joue dans le paysage littéraire de l'époque.

7 Le lac, tout comme la rivière, est un élément majeur de la composition des grands jardins paysagers du dernier quart du siècle des Lumières. Parmi les parcs aristocratiques les plus fameux figurent ceux d'Ermenonville, du Raincy ou de Méréville. Jeux de paysages, surprises visuelles et auditives, les lacs touchent les sentiments et les sens. Jean-Marie Blondel, auteur reconnu sur l'art des jardins, publie en 1776 La Théorie des jardins où il résume les qualités accordées aux effets aquatiques : 
Les eaux sont au paysage ce que l'âme est au corps : elles animent une scène, en donnent de l'éclat à une perspective et répandent de la fraîcheur et de la vie dans tous les lieux où elles se trouvent. Par leur charme, elles attirent nos regards et fixent nos attentions.

8 Pour parvenir à de tels effets, les aménagements nécessitent alors de véritables prouesses en matière de terrassement et de maitrise des techniques hydrauliques. Recompositions de la nature tout comme les rivières qui les accompagnent, les lacs restent, jusqu'à l'apparition des grands parcs publics parisiens du milieu du $\mathrm{XIX}^{\mathrm{e}}$ siècle, l'apanage d'une élite sociale et financière.

9 Au-delà de cette nature lacustre recomposée, le lac en tant qu'objet de paysage naturel est également, dès la fin $\mathrm{du}_{\mathrm{XVIII}}^{\mathrm{e}}$ siècle et au début $d u$ XIX ${ }^{\mathrm{e}}$, un sujet de contemplation qui annonce l'approche romantique de la nature.

10 Parmi les auteurs qui ont promu la dimension poétique du lac et des îles, il faut sans conteste citer Jean-Jacques Rousseau dans ses écrits pré-romantiques. À maintes reprises, le philosophe vante les lacs comme des lieux propices à la méditation, avec une prédilection pour le lac de Genève :

L'aspect du lac de Genève et de ses admirables côtes eut toujours à mes yeux un attrait particulier, que je ne saurois expliquer, et qui ne tient pas seulement à la beauté du spectacle, mais à je ne sais quoi de plus intéressant qui m'affecte et m'attendrit ${ }^{3}$.

11 C'est d'ailleurs sur ce lac qu'il situe l'action de La Nouvelle Héloïse 4 . Dans la cinquième promenade des Rêveries du promeneur solitaire, il évoque aussi le lac de Bienne avec l'île Saint-Pierre, près de Neuchâtel :

Quand le soir approchait je descendais des cimes de l'île et j'allais volontiers m'asseoir au bord du lac dans quelque asile caché; là le bruit des vagues et l'agitation de l'eau fixant mes sens et chassant de mon âme toute autre agitation la plongeaient dans une rêverie délicieuse où la nuit me surprenait souvent sans que je m'en fusse aperçu ${ }^{5}$.

12 Fait moins souvent mentionné, le philosophe a également entretenu des liens privilégiés avec le lac d'Enghien. En effet, en 1756, fuyant les miasmes et fumées de Paris, il trouve asile à Montmorency au lieu-dit «L'Ermitage » puis dans la maison du Petit Mont-Louis. C'est sur ce promontoire dominant le lac qu'il compose ses œuvres majeures dont Julie ou la Nouvelle Héloïse, Le Contrat social et Émile ou de l'Éducation. D'autre part, nous savons qu'il donnait des leçons de botanique à Madeleine Gauthier, fille de Mme Delessert, famille avec laquelle il entretenait des relations amicales... Madeleine Gauthier est celle qui, en 1803, acquiert l'autorisation d'exploiter les eaux d'Enghien'.

13 La présence de Rousseau est décisive pour la notoriété d'Enghien, rappelée fréquemment dans des ouvrages de nature très différente, guides ou romans. En témoigne Jules Lecomte, auteur aujourd'hui bien oublié, qui écrit dans Le capitaine Sabord: " On sait combien Montmorency est un charmant village plein de souvenirs du grand philosophe, son bois si mélancolique semble le jardin d'une grande villa dont le lac d'Enghien est le miroir 7 . » Pour la promotion de la station thermale, se construit un véritable mythe autour de «l'auteur de l'Émile ». Le site est présenté comme un «lieu de rêverie philosophique à la réflexion, à l'enthousiasme secret qui engendre les grandes idées »... En 1826, le journal La Quotidienne ${ }^{8}$ évoque les illustres présences qui en ont fait son histoire : «Allez visiter Enghien et le lac qui virent souvent errer sur ses bords Catinat et Jean-Jacques Rousseau. » Dans l'un des guides les plus fameux de la 
station, celui du docteur Révillé-Parisé publié en 1842, le malade est assimilé à « un promeneur ", "au philosophe rêveur" qui, imprégné du souvenir des Rêveries du promeneur solitaire, peut déjà progresser vers la guérison. Le site est présenté comme «la terre chérie » de "l'auteur de l'émile » qui " pourtant né près des Alpes » aimait tant à y «herboriser». «La solitude des lieux champêtres» qui engendre «la profondeur de la réflexion ", est évoquée, encore une allusion à Rousseau... Mais si la référence rousseauiste est ici incontestable, il faut néanmoins souligner que le goût de la solitude et de la promenade sont fréquemment invoqués au titre des pratiques des stations thermales du milieu du XIXe siècle. Citons, parmi les nombreux exemples, la déambulation champêtre des curistes vichyssois ${ }^{9}$ sur les bords de l'Allier. Révillé-Parisé évoque d'ailleurs dans son guide l'une des célèbres baigneuses de la station thermale de Vichy, Madame de Sévigné, et emprunte à sa plume pour constater l'importance du lieu sur la guérison : «Le pays seul me guérirait » disait la marquise, confortant la théorie des médecins sur l'importance " des soins, des airs et des lieux ».

Enfin, dans les années 1820, on ne peut évoquer le lac d'Enghien sans faire référence à Lamartine, qui dans son poème Le lac de B..., immortalise le lac du Bourget bordant les rives d'Aix-les-Bains, autre lieu de villégiature et de thermalisme :

Qu'il soit dans ton repos, qu'il soit dans tes orages,

Beau lac, et dans l'aspect de tes riants coteaux,

Et dans ces noirs sapins, et dans ces rocs sauvages

Qui pendent sur tes eaux ${ }^{10}$ !

\section{Un village autour d'un lac : le projet de Péligot}

En 1825, le calme de la belle nature et la possibilité, rare, de se trouver si près de Paris "sur une grève solitaire $»^{11}$ caractérisent Enghien. Peu de temps après, le docteur Longchamp $\mathrm{p}^{12}$ décrit un

[...] village situé au bord du bel étang de Saint Gratien ${ }^{13}$ avec un bâtiment thermal vaste et agréablement situé entouré de belles habitations [...] le nouvel encaissement que l'on vient de faire à l'étang qui ne laissera plus sur ses bords pendant l'été des vases [...] permettra encore de l'entourer de jolies maisons qui seront recherchées par les habitants de la capitale qui voudront respirer un air salubre dans une contrée où il $\mathrm{y}$ a de la verdure et de l'eau, choses si rares aux environs de Paris.

Le développement potentiel d'un village autour du lac et de la station thermale ne manque pas d'intéresser Jean-Baptiste Péligot (1777-1837) qui en 1821, abandonne son poste d'administrateur en chef des hôpitaux et hospices de Paris pour se consacrer au lancement de la station d'Enghien. Il est considéré comme « le fondateur de la colonie d'Enghien ». Ayant acquis auprès du comte de Luçay ${ }^{14}$, dans les années 1825-1829, le domaine de Saint-Gratien et le lac, il lance une grande opération de lotissement, divisant l'ensemble en 300 parcelles pour «que de charmantes maisons de campagne y soient créées à grands frais par des acquéreurs ${ }^{15}$ ». Le lac est endigué sur son pourtour et plusieurs puits artésiens sont forés pour l'alimenter. Ce lac, d'un kilomètre de long, et d'une superficie de 46 hectares, présente un site dont la beauté est sans nul doute l'un des facteurs de l'essor de la station. 
Figure 2

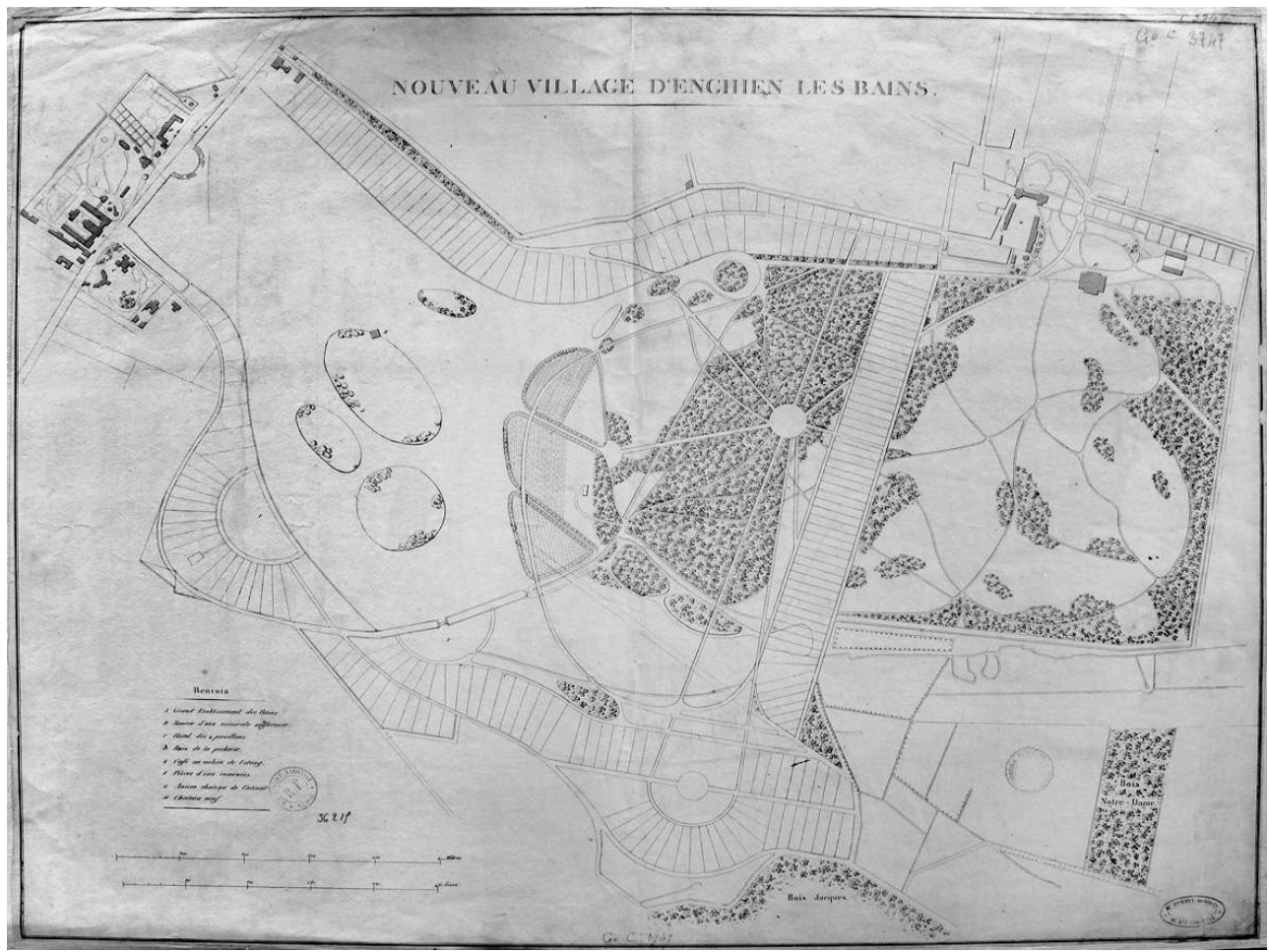

«Plan du petit village d'Enghien », vers 1829. BnF cartes et Plans.

Repro. Vialles, Jean-Bernard. (c) Conseil régional d'Île-de-France, service Patrimoines et Inventaire, 2010. (base Mémoire : 20109500057)

17 Conservés à la Bibliothèque nationale ${ }^{16}$ et portant pour l'un le cachet de l'architecte Dommey, les plans du «Nouveau village d'Enghien-les-Bains» font état de ce projet (fig. 2). Les seuls édifices existants et portés sur ces documents sont le grand bâtiment thermal, les thermes construits par l'architecte Constantin et l'hôtel des Quatre Pavillons (fig. 3) auprès duquel se trouve la première maison de la station ${ }^{17}$. Le lac n'a pas alors sa configuration actuelle et comprend quatre îles dont l'une est pourvue d'un café. Plusieurs témoignages mentionnent cette destination de promenade en bateau, café circulaire, « café lacustre sur pilotis ${ }^{18}$. 
Figure 3

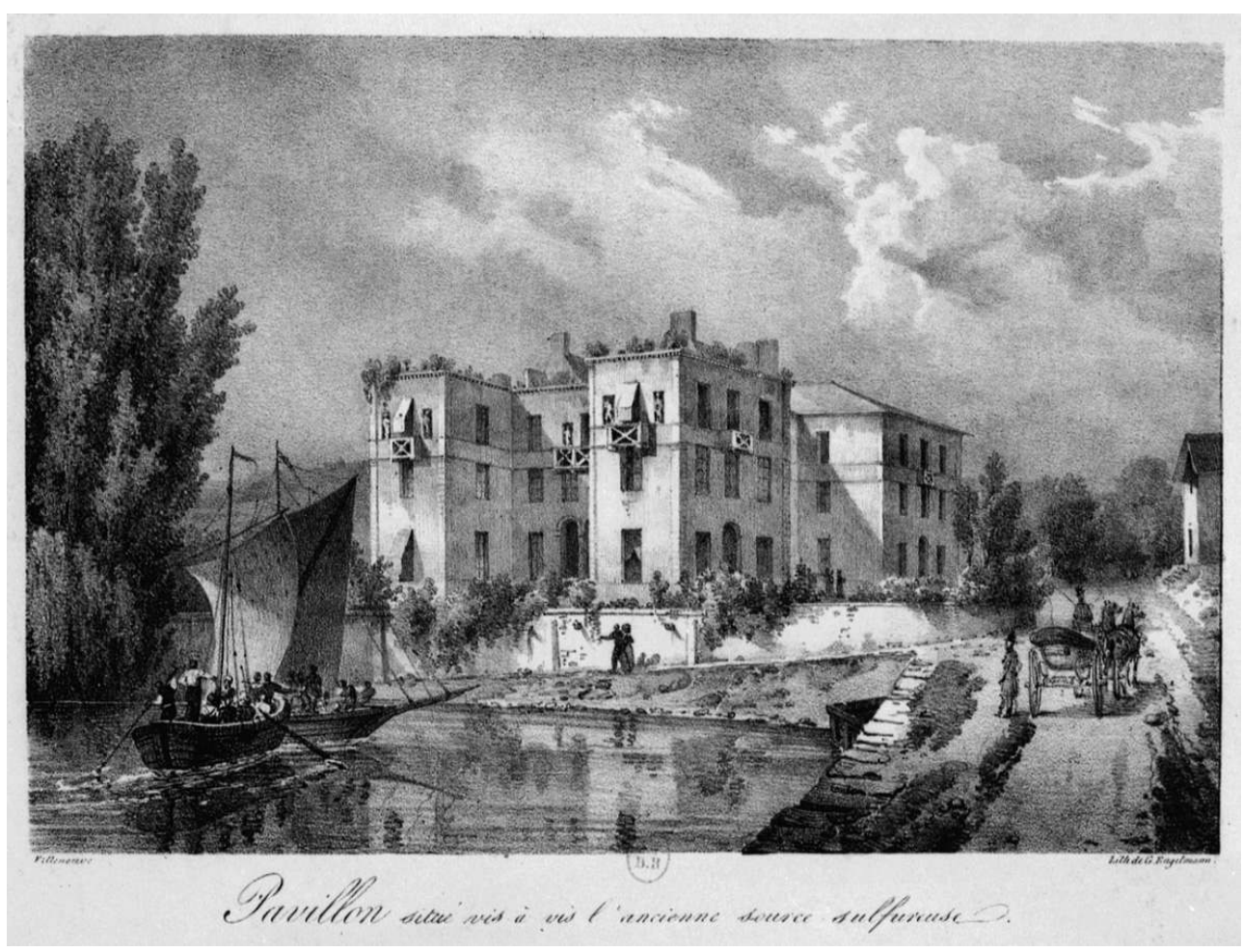

L'hôtel des Quatre Pavillons, gravure vers 1830. BnF Estampes, topo Va, Fol Va, tome 3, Val-d'Oise. Phot. Vialles, Jean-Bernard. (c) Conseil régional d'île-de-France, service Patrimoines et Inventaire, 1980. (base Mémoire : 1980950896Z)

Ces plans, inédits jusqu'à notre étude, constituent un témoignage éclairant sur l'histoire de l'urbanisme de la villégiature en France et tout particulièrement celle de l'île-de-France. Au nombre des premières « colonies » de villégiature de grande taille des environs de la capitale, elle précède de peu celle de Maisons-Laffitte en 1833, suivie par la colonie du Vésinet en 1856, pour ne citer que les plus fameuses ${ }^{19}$. Mais le projet, sans doute trop ambitieux pour être maintenu après la faillite de Péligot, n'aboutit pas dans sa totalité ${ }^{20}$. Il était pourtant prometteur : une succession de parcelles laniérées était disposée sur le pourtour du lac, zone constituant les actuelles avenues de Ceinture et du Lac, et la répartition des lots se prolongeait sur une grande bande rectiligne à l'intérieur des terres, entre deux parcs. L'ensemble du lotissement était dessiné entre lac et parcs, dans une réelle intention paysagère. En plusieurs points, le tracé s'organisait en de savants agencements en hémicycle, formant des places, ou même parfois des bassins en demi-lune sur lesquels aboutissaient des parcelles en éventail.

La redondance de ces formes demi-circulaires, motif urbain d'outre-Manche, laisse à penser que ce plan, très novateur pour l'époque, est inspiré des modèles anglais. On peut évoquer l'expérience contemporaine du " nouveau village d'Orléans ", établi entre 1827 et 1834 à proximité de l'enceinte des Fermiers généraux, où la référence anglaise est clairement énoncée dans la présence d'un square entouré d'une sorte de crescent avec une fontaine au centre, le tout clos par une grille ${ }^{21}$. Plus évocateurs encore de la réelle influence anglaise sur le dessin d'Enghien sont les projets anglais tels ceux, à Londres, de l'aménagement du domaine Eyre dans le quartier de St Johns Wood dès 1794, celui de Marylebone Park par John White en 1809 pour le futur Regent's Park ${ }^{22}$ ou, 
plus tardive, la réalisation de Victoria Park en 1837, lotissement de ville à la campagne qui permettait aux habitants de Manchester d'en fuir les fumées ${ }^{23}$.

Péligot avait également conçu un cahier des charges afin de conserver un cadre naturel au site malgré l'implantation de maisons. Il est interdit de se clore du côté des berges, obligation compensée par le privilège d'un droit à la promenade sur le lac accordé aux propriétaires. Un alignement fixe la disposition du bâti « à 20 mètres de distance l'un de l'autre » et l'on ne peut, "construire aucun bâtiment incommode ou nuisible aux propriétaires des lots, ni introduire pour les bâtiments à construire ou dans leurs dépendances des gens dont l'exercice journalier occasionne du bruit ${ }^{24} »$. Dernier point, inhérent aux qualités thermales du lieu, il est spécifié que l'on ne "peut faire usage des eaux sulfureuses qui seraient découvertes sur les terrains vendus " accordant ainsi un "périmètre de protection" à l'exploitation thermale ${ }^{25}$. En contrepartie de ces contraintes, Péligot et sa société s'engagent à créer « le jardin de l'embarcadère pour l'agrément et l'aspect le plus agréable avec aucune construction ni mur à cet endroit ». L'acte de création de ce lotissement est relaté dans le Tour de la vallée, guide édité en 1856, comme un événement surnaturel :

Péligot veut que cent villas élégantes s'élèvent bientôt, comme par enchantement, au milieu de la riante vallée de Montmorency; et de même que Moïse a frappé la mer Rouge avec sa baguette pour sauver les Hébreux, de même, le successeur de Veillard, pour peupler un charmant désert, s'adresse premièrement au lac en lui disant: “Tu n'iras pas plus loin! L'étang d'Enghien est encaissé et entouré d'une large avenue ${ }^{26}$.

Parmi les premiers villégiateurs des bords du lac, amateurs de retraites campagnardes, figurent plusieurs artistes parisiens dont les plus illustres sont les peintres Eugène Isabey, Paul Delaroche, Horace Vernet ou Charles Giraud, le sculpteur James Pradier mais aussi les actrices en vogue telles Mlle Mars ou Mlle Ozy (fig. 4). En 1847, sur la centaine de maisons existantes dans le hameau d'Enghien, quarante-trois maisons de plaisance ou «campagnes » sont la propriété de villégiateurs, situées pour la plupart sur les bords du lac. Les témoignages contemporains parlent alors "d'un véritable village ». 
Figure 4

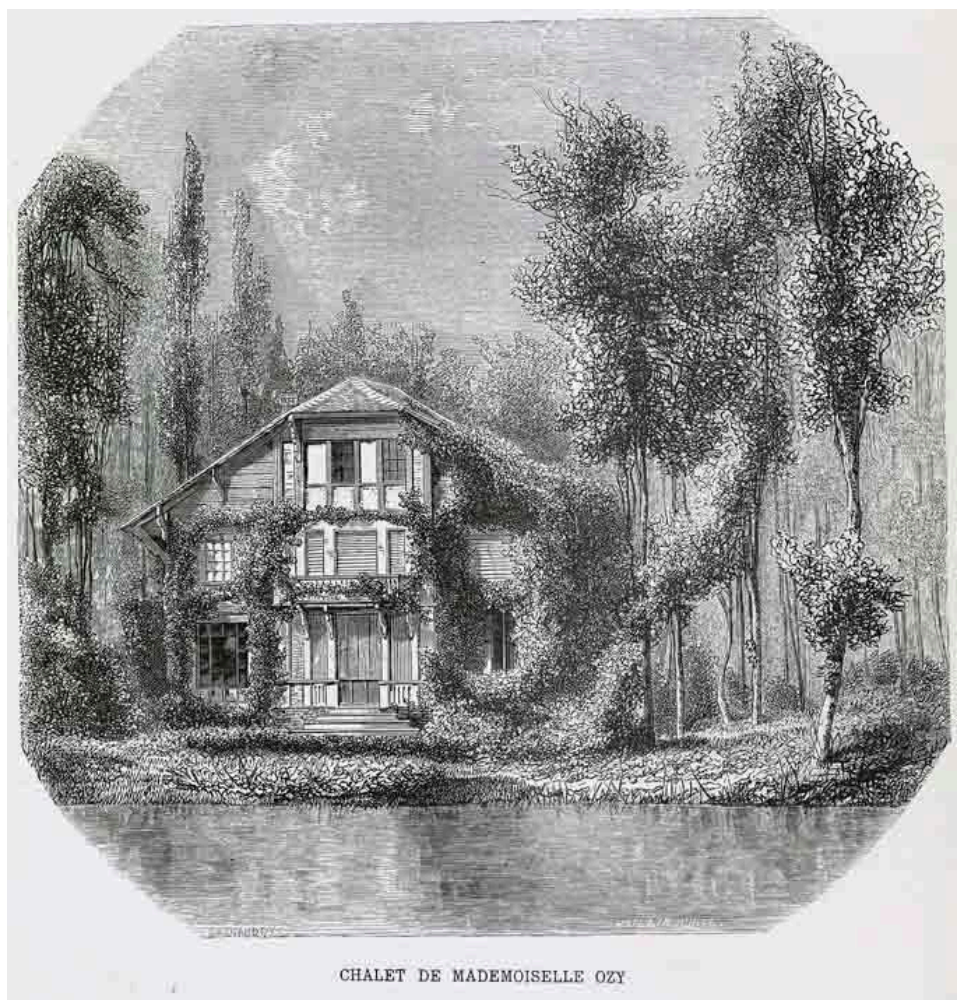

Maison de Mademoiselle Hozy, gravure extraite d'Enghien et ses environs par Émile de Girardin et alii. Paris : Michel Levy frères, 1860.

Phot. Vialles, Jean-Bernard. @ Conseil régional d'Île-de-France, service Patrimoines et Inventaire, 2009. (base Mémoire : 2009500394)

\section{L'évasion à deux pas de la capitale}

22 L'image du lac d'Enghien au cours du XIX ${ }^{e}$ siècle trouve son expression dans nombre d'effets littéraires et musicaux ${ }^{27}$ et ceci grâce à des références qui renvoient aux plus fameux paysages européens. Le lac reste l'élément essentiel de la promotion du site et fait l'objet d'une abondante iconographie (fig. 5). 
Figure 5

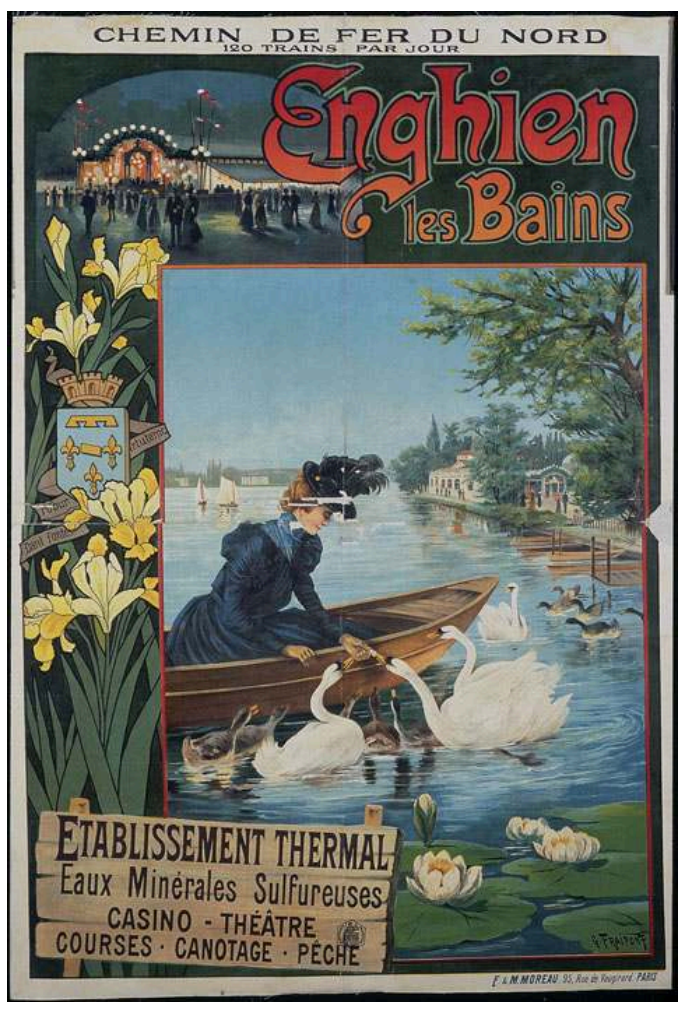

Le lac d'Enghien, affiche de la Cie du chemin de fer du Nord. Musée de l'île-de-France.

Repro. Vialles, Jean-Bernard. (c) Conseil régional d'Île-de-France, service Patrimoines et Inventaire, 2006. (base Mémoire : 2006950076)

Ce qui prévaut tout d'abord, c'est la proximité du lieu avec Paris. Cet «Éden des villégiatures élégantes de la capitale ${ }^{28}$ » - « on y est à la fois si près et si loin de Paris ${ }^{29}$ " - et son lac sont « une des merveilles de la banlieue de Paris [...] où règne un collier de villas délicieuses, la plupart construites en forme de chalets suisses, d'autres en petites maisons gothiques, se mirant dans les eaux paisibles ${ }^{30}$ » (fig. 6). Au même titre que les jardins des Tuileries, du Palais Royal, les Champs-Élysées, Longchamp, Saint-Cloud, ou les tout récents aménagements des bois de Boulogne et de Vincennes, Enghien figure au nombre des plus belles promenades parisiennes ${ }^{31}$. Dans son poème Venise au lac d'Enghien, Léon Duvauchel considère que si, dans l'espace d'un dimanche, un Parisien veut découvrir Venise, c'est à Enghien qu'il doit se rendre ${ }^{32}$ ! Dans une habile tournure littéraire, Émile de Girardin transforme en qualité ce qu'il présente en défaut...

Bade, le Tréport, Biarritz, Vichy, Monaco, Spa, tout ce qu'on est convenu d'appeler les Bains et les Eaux à la mode, n'offrent à leurs hôtes rien de plus gracieux que la vallée de Montmorency. Enghien n'a qu'un défaut, c'est d'être à vingt minutes de Paris $^{33}$. 


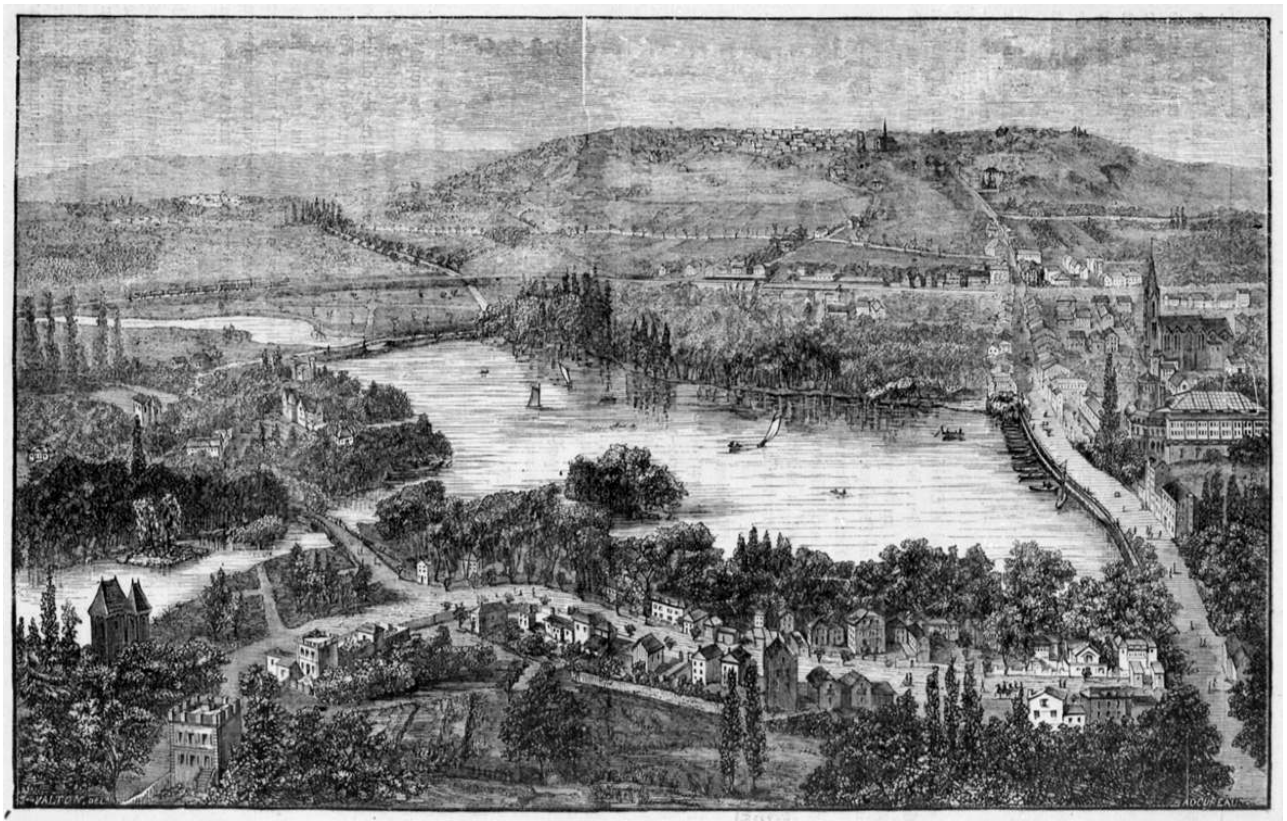

Vue panoramique d'Enghien-les-Bains ; le lac et la ville dominés par les hauteurs de Montmorency, troisième quart du xix ${ }^{\mathrm{e}}$ siècle, lithographie de Georges Muller. BnF Estampes, topo Va, Fol Va, tome 3, Val-d'Oise.

Repro. Vialles, Jean-Bernard. @ Conseil régional d'Île-de-France, service Patrimoines et Inventaire, 1980. (base Mémoire : 1980950915Z)

Le Guide d'Enghien et ses thermes, en $1868^{34}$ surenchérit :

À quelques kilomètres de Paris se trouve Enghien, l'un des plus jolis pays du monde. Son beau lac, dont les rives sont parsemées de coquettes villas et d'arbres majestueux qui se réfléchissent dans ses eaux limpides, est d'un effet magique surtout aux derniers rayons du soleil [...] L'on va chercher bien loin, dans les Pyrénées ou ailleurs, ce qu'on a sous la main : climat heureux, pays magnifique, bois admirables, eaux dont la puissance n'a point de rivale parmi toutes les eaux sulfureuses de France.

La magie et le surnaturel que les guides accordent autant au climat qu'au paysage sont des arguments publicitaires inhérents à la production des guides de cette période. Mais une analyse plus fine de ces lignes peut également conduire à d'autres considérations. En effet, certains propos conduisaient le lecteur vers des références littéraires à ce jour oubliées: "Il semble qu'une fée bienfaisante vous a transporté dans une de ces îles fortunées dont on lit avec plaisir la brillante description de certains voyageurs [...] un site délicieux, un lac d'une étendue proportionnée au paysage ${ }^{35}$. » Sans doute sont évoquées là les descriptions d'îles et de paysages d'ouvrages célèbres toujours en vogue. En 1719, Daniel Defoe publie Robinson Crusoé dont la première traduction française, la plus connue, est réalisée en 1836 par le poète et écrivain Pétrus Borel ${ }^{36}$, alors que le village d'Enghien en est encore à ses prémices ${ }^{37}$. Ce récit, reprenant la véritable histoire du marin écossais Alexandre Selkirk, alimente l'imaginaire et le désir de rivage et d'insularité inhérents à la nature humaine ${ }^{38}$. Rappelons également que Paul et Virginie, publié en 1788 par Jacques-Henri Bernardin de Saint-Pierre, grand succès de librairie de la fin du XVIII et du XIX siècle, a aussi contribué pour une large part au mythe de l'île arcadienne ${ }^{39}$. 
26 Fait notable pour notre propos, en 1880, Henri-Honoré Bescherelle, dans son Dictionnaire classique de la langue française le plus exact et le plus complet de tous les ouvrages de ce genre, définit ainsi le lac: "grande étendue d'eau environnée par les terres ", citant comme exemples les lacs de Genève, de Constance, de Côme et d'Enghien. Comment a-t-il été possible d'établir comme une base quasi scientifique ce qui n'a été forgé que par des écrits dont le caractère dithyrambique n'avait somme toute qu'une finalité publicitaire ou romanesque ? Avec le site d'Enghien, il semblerait que la réalité se soit emparée de la fiction! Les raisons en sont diverses. La proximité des hauteurs de Montmorency, qui donnent au lac un paysage "montagneux", a suscité des comparaisons avec les sites italiens et français les plus fameux pour affirmer qu'il est plus simple de se rendre à Enghien : "Où est donc situé ce pays ? En Italie ? Au-delà des Pyrénées? Sur les bords du Gange? Point du tout, on le trouve un peu plus loin que Saint-Denis, à quelques lieues de la barrière. » Le même guide, qui n'est autre que celui de Révillé-Parisé ${ }^{40}$, raille ceux qui partent au loin pour voir le mont Blanc, la Jungfrau ou le pic du Midi alors que l'on a aux portes de Paris une vallée et un lac qui offrent également des trésors de la nature "mère de toute poésie ». De manière récurrente, le lac d'Enghien est mis en parallèle, bien sûr à son avantage, avec les lacs européens les plus illustres :

Véritable miniature des grands lacs de la Suisse [...] si la vue de ce lac est moins magnifique [...] que celle des lacs que renferment les Alpes en revanche on y craint ni les bourrasques, ni la fureur subite des vagues [...] on peut hardiment y naviguer tout le temps et en toute saison sur tous les points de ses plages verdoyantes ${ }^{41}$.

Les maisons d'Enghien sont comparées à celles des bords du lac de Côme, le plus renommé d'Italie avec le lac Majeur et le lac de Garde, présenté par Stendhal comme "l'un des plus beaux sites du monde». La confusion des genres se veut telle qu'un guide décrit une maison comme « une villa des bords du lac de Côme transplantée sur les bords du lac d'Enghien ${ }^{42}$ ». Au-delà des guides, la littérature renforce les analogies. Sans aucunement prétendre à l'exhaustivité mais en respectant un choix chronologique, citons en 1859 La Sœur de Moïse qui, à propos d'Enghien, souligne avec humour " qu'aujourd'hui on va au lac de Genève en pantoufle ${ }^{43}$ ", Théophile Gauthier, qui considère, dans la préface d'un recueil de poésies d'Auguste Châtillon, que l'on va à Enghien « à défaut du lac d'Elvire ou du lac Majeur », ou Théodore de Banville, qui situe son héroïne des Parisiennes en promenade du soir «sur le lac d'Enghien ou le lac de Côme [...] on la rencontre échevelée [...] preuve certaine qu'elle a trop lu Lamartine $»^{44}$.

Paradoxe de cette notoriété, Enghien à son tour devient une référence! En 1862, dans Les Choses du présent, Edmond Texier évoque la rive du Rhin de Schaffhouse près du lac de Constance comme « une Suisse coquette attifée en corset [...] jolie comme le lac d'Enghien $»^{45}$. Ibert Wolff, dans Les Mémoires du Boulevard publiées en 1866, considère que le Rhin ne sera bientôt plus qu'une succursale du lac d'Enghien, regrettant la disparition de petits villages pittoresques qui font place à des habitations modernes et coquettes qu'il compare à celles de la station parisienne. En 1887, Maurice Jametel, dans Pékin, souvenir de l'Empire du Milieu, décrit « des jardins et élégantes habitations aux plates-bandes bien entretenues [...] qui vous rappellent bien plus les élégantes villas de Kiew et d'Enghien que le pays des clochettes et des paravents ${ }^{46}{ }^{\prime \prime}$.

Plus étonnant encore, l'auteur des Chasses dans l'Amérique du Nord ${ }^{47}$ compare un grand lac canadien à celui d'Enghien, de même que dans À travers le royaume du Tamerlan, en 1892, une visite de la ville russe de Petropavlosk nous ramène vers ces rivages : «Un petit lac bordé de villas enfouies dans la verdure et rappelant le lac d'Enghien et ses 
environs $s^{48}$. On ne saurait achever ce tour des visions enghiennoisess sans évoquer Le Portfolio colonial de John L. Stoddard décrivant en 1895 les paysages, villes et industries des possessions et dépendances françaises où le lac de Wesley égale le lac d'Enghien! La représentation d'Enghien comme modèle de ville pittoresque est si forte dans les esprits contemporains qu'un Parisien allant à Bonn au milieu de «chalets suisses, de maisons peintes, de jardins anglais, d'habitations en carton pierre qui bordent les rues » dira : « C'est Enghien moins le lac ${ }^{49} » .$.

Dans la surenchère qui a contribué à la notoriété du site, c'est enfin l'image de la mer qui a été saisie : «la petite mer d'Enghien ${ }^{50}$. Cette comparaison entre lac et mer est une constante dans l'histoire littéraire des lacs, mais elle est d'autant plus insolite que celui d'Enghien est de taille modeste. Pourtant, plusieurs romans la reprennent: Le Vengeur du mari, en 1862, cite le lac comme «cette mer de poche que les Parisiens appellent le lac d'Enghien ${ }^{51}$ ». Le roman est suivi la même année de la publication des Aventures d'un petit Parisien où le jeune héros à qui l'on demande s'il a déjà vu la mer répond « d'un air dédaigneux [...] j'ai vu le lac d'Enghien, ce qui est à peu près la même $\operatorname{chose}^{52} »$.

\section{La présence du lac a-t-elle eu une influence sur son architecture?}

Figure 7

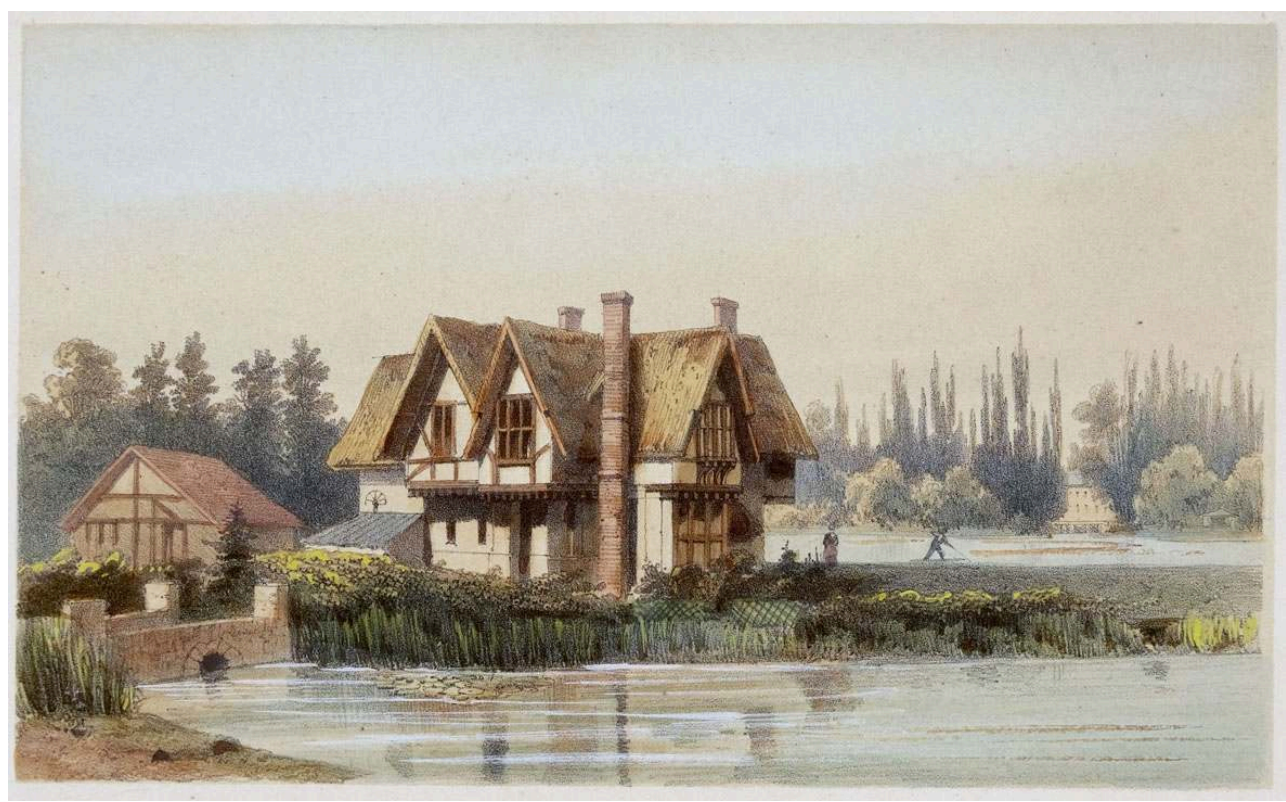

Chaumière au bord de l'eau, chromolithographie par Georges Muller, Paris et ses environs, deuxième quart du xix siècle. Musée de l'île-de-France.

Repro. Vialles, Jean-Bernard. (c) Conseil régional d'Île-de-France, service Patrimoines et Inventaire, 2006. (base Mémoire : 2006950070)

Au-delà des équipements thermaux qui, au cours du temps ajustent leur architecture aux modes et aux exigences médicales, les premiers monuments de la station sont les maisons. Implantées pour les premières autour du lac pour jouir au mieux du paysage, elles adaptent leur morphologie à l'originalité du site par leur caractère pittoresque. 
Durant les premières années, l'architecture est aux chalets, ermitages et chaumières (fig. 7), puis aux maisons de "genre» gothique, Renaissance ou néo-normand, complétant ce mélange des styles que les contemporains admiraient pour leur éclectisme. Les façades les plus ouvragées avec balcons et terrasses sont orientées vers la vue et donc vers le lac. Durant la deuxième moitié du XIX ${ }^{\mathrm{e}}$ siècle, plusieurs belvédères (fig. 8) apparaissent, réponse architecturale aux attraits du site, autant vers le lac que vers les hauteurs de Montmorency. Tour possédant une pièce pour jouir de la vue, cet élément est une caractéristique classique de l'architecture de la villégiature.

\section{Figure 8}

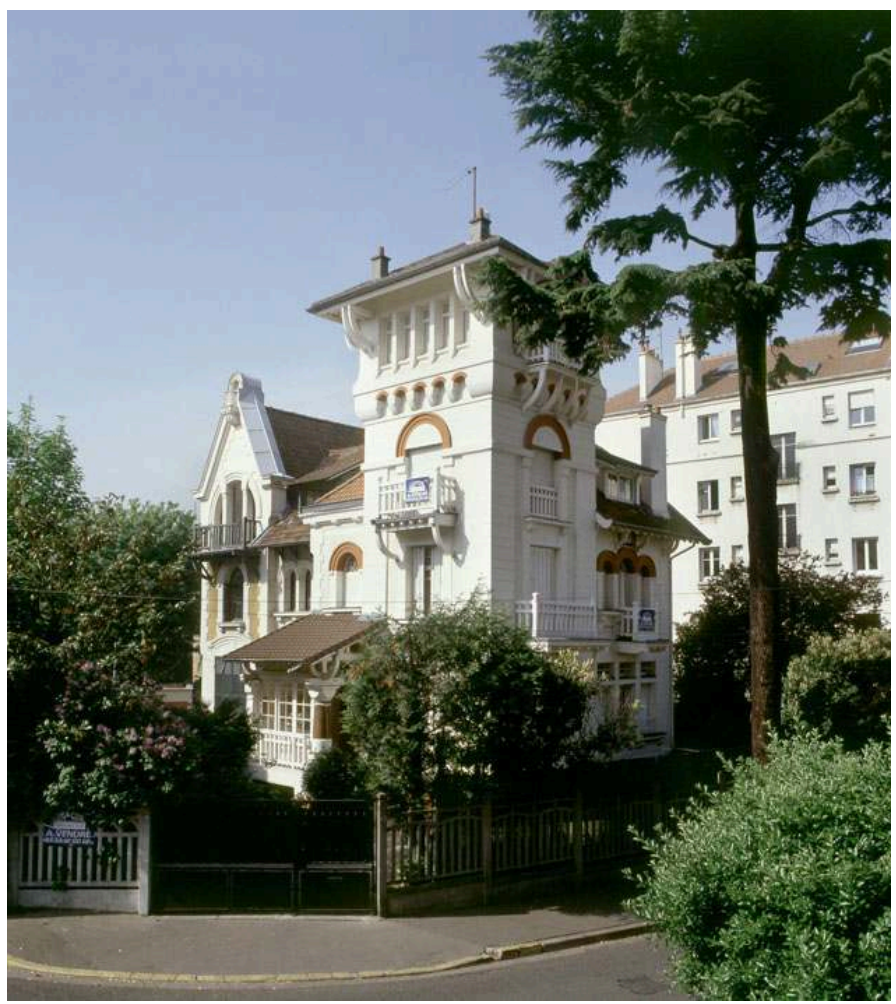

Le Rêve, villa avec belvédère qui à l'origine avait vue sur le lac.

Phot. Vialles, Jean-Bernard. (c) Conseil régional d'île-de-France, service Patrimoines et Inventaire, 2012. (base Mémoire : 2006950212XA)

Autre élément fréquent sur les rivages, des embarcadères et des kiosques ponctuaient les bords du lac d'Enghien. Cette architecture trop fragile a malheureusement disparu et nous la connaissons essentiellement par des gravures et des cartes postales. Le seul embarcadère subsistant est aujourd'hui conservé sur le terrain du lycée GustaveMonod, ancienne propriété dite du château Léon : deux lions sculptés en cantonnent l'accès (fig. 9). 
Figure 9

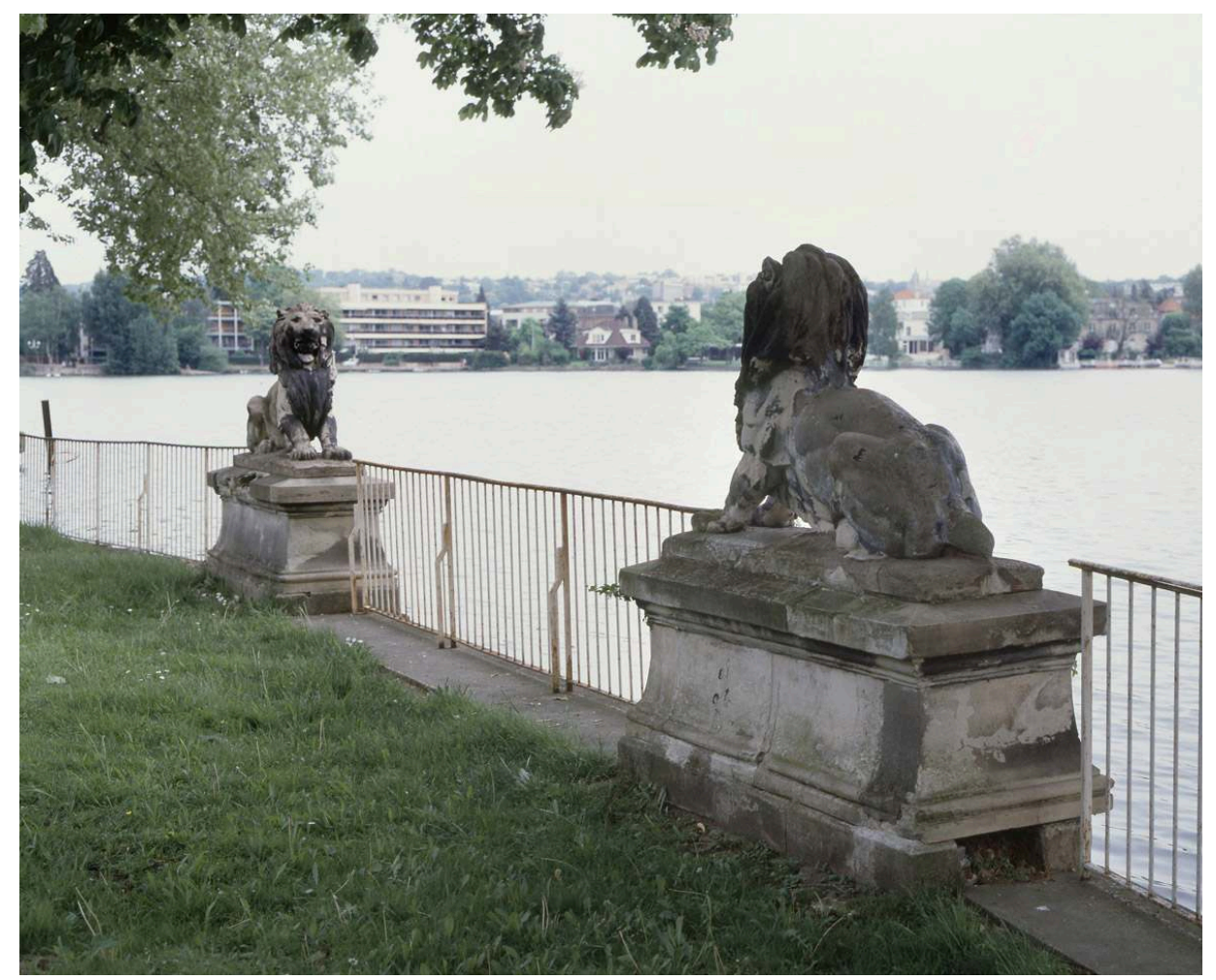

Embarcadère du château Léon, actuel lycée Gustave-Monod, encadré par deux lions de terre cuite. Phot. Vialles, Jean-Bernard. (c) Conseil régional d'Île-de-France, service Patrimoines et Inventaire, 2006. (base Mémoire : 2006950101XA)

Mais c'est sur la digue et dans ses abords que les aménagements les plus spécifiques se mettent en place. Dès l'implantation du premier village, la digue située devant les établissements thermaux est un espace réservé qui peu à peu est organisé. Un grand projet de kursaal devait prendre place sur le lac au bout d'une jetée, projet interrompu par la guerre de 1870. Cette idée audacieuse reprenait celle tout récemment expérimentée par les Anglais en 1863-1866 à Brighton. Une société avait été créée pour réunir les fonds nécessaires et l'architecte A. Ponsin avait dessiné un superbe établissement dont le répertoire des formes propres à la tradition architecturale orientaliste se faisait l'écho de celle qui se développait alors en France dans les stations de villégiature. L'ensemble devait être inauguré en 1867 pour accueillir les visiteurs de l'Exposition universelle (fig. 10). Une touche d'exotisme fut toutefois maintenue avec l'installation d'un grand pavillon chinois comportant un café, au bord du lac, au bout de la jetée. 
Figure 10

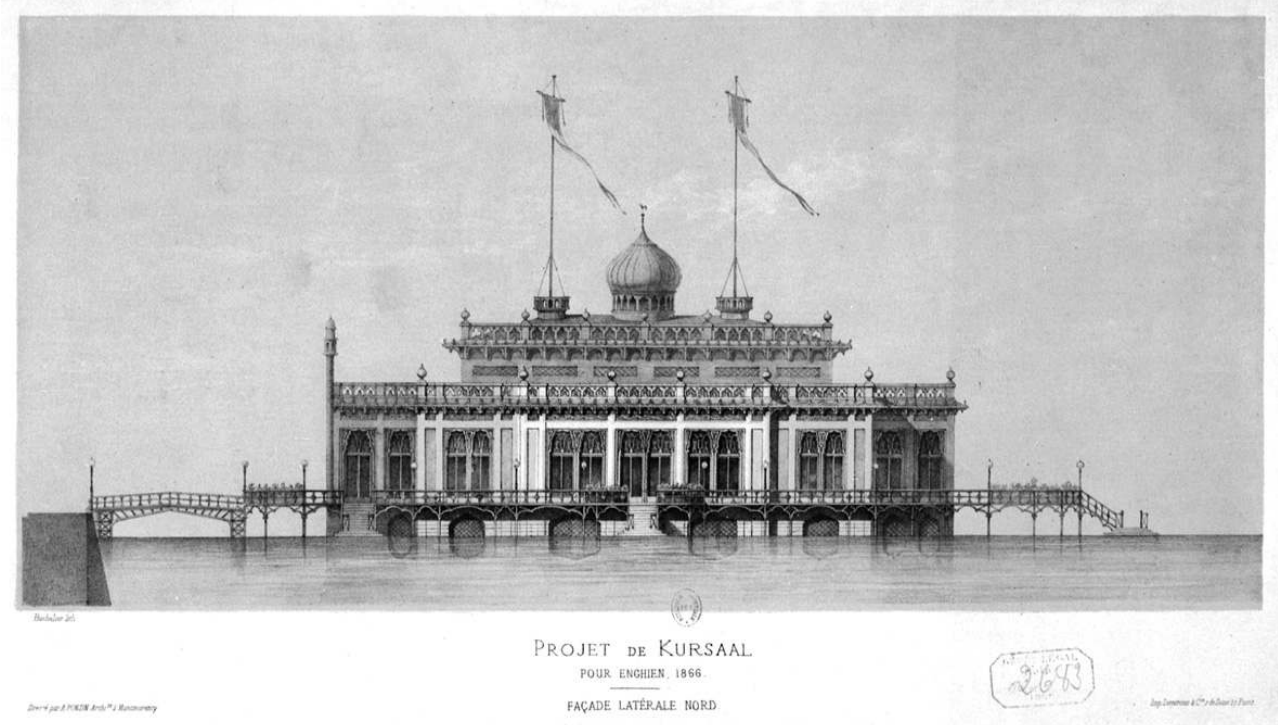

Élévation latérale du kursaal, projet par A. Ponsin, 1866. BnF Estampes, topo Va, tome 3, Val-d'Oise Repro. Vialles, Jean-Bernard. (c) Conseil régional d'île-de-France, service Patrimoines et Inventaire, 1980. (base Mémoire : 1980950861Z)

Figure 11

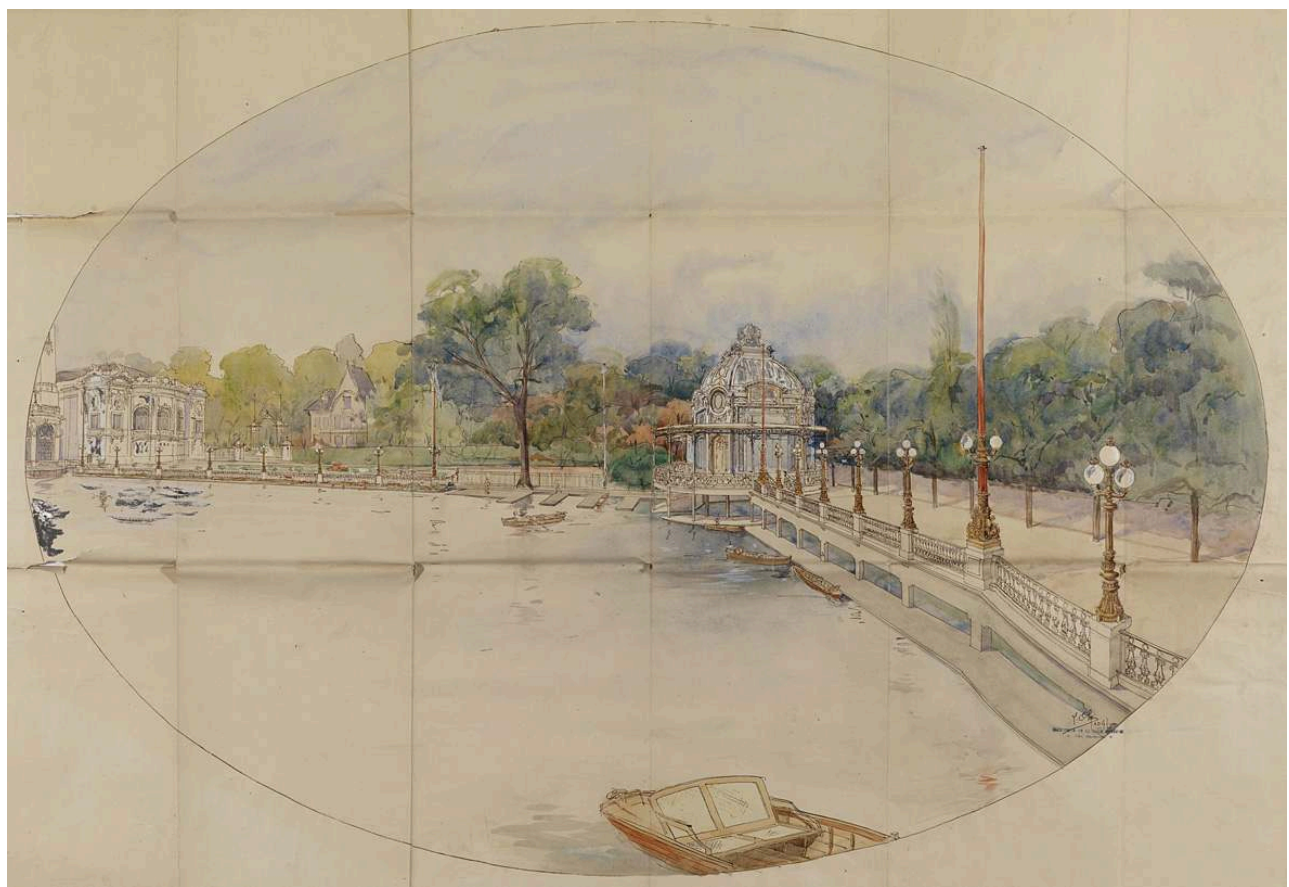

Projet de l'estacade, 1910, Louis Olivier. Archives communales d'Enghien-les-Bains

Repro. Vialles, Jean-Bernard. (c) Conseil régional d'Île-de-France, service Patrimoines et Inventaire, 2009. (base Mémoire : 20099500143)

Bords de mer, bords de lac sont le lieu principal de déambulation des baigneurs et villégiateurs. Enghien possède sa jetée, aménagée en 1910. Véritable vitrine de la station, elle a été conçue par l'architecte Louis Olivier sur commande de la 
municipalité. Grande estacade de 246 mètres de long, elle s'inscrit dans les usages des stations balnéaires comme la grande jetée-promenade d'Arcachon, inaugurée en 1903 (fig. 11). Ces lieux voient également l'implantation de casinos, comme ceux des stations thermales de Bagnoles-de-l'Orne, Évian et Enghien. L'architecte Édouard Autant est l'auteur de l'un des édifices les plus audacieux du corpus. Construit en 1901-1902, il est en forme de navire, amarré sur les berges du lac d'Enghien. Ce casino-navire (fig. 12), construit très rapidement, ne résista guère au temps et fut remplacé en 1908 par un nouvel édifice. La qualité du site, sa proximité avec Paris, font que d'autres projets de casinos sont lancés sur les bords du lac, tel le Windsor Casino, dont la façade de soixante-dix mètres de long, dessinée en 1909 par les architectes Boët et Roset, devait s'élancer face à la jetée (fig. 13).

Figure 12

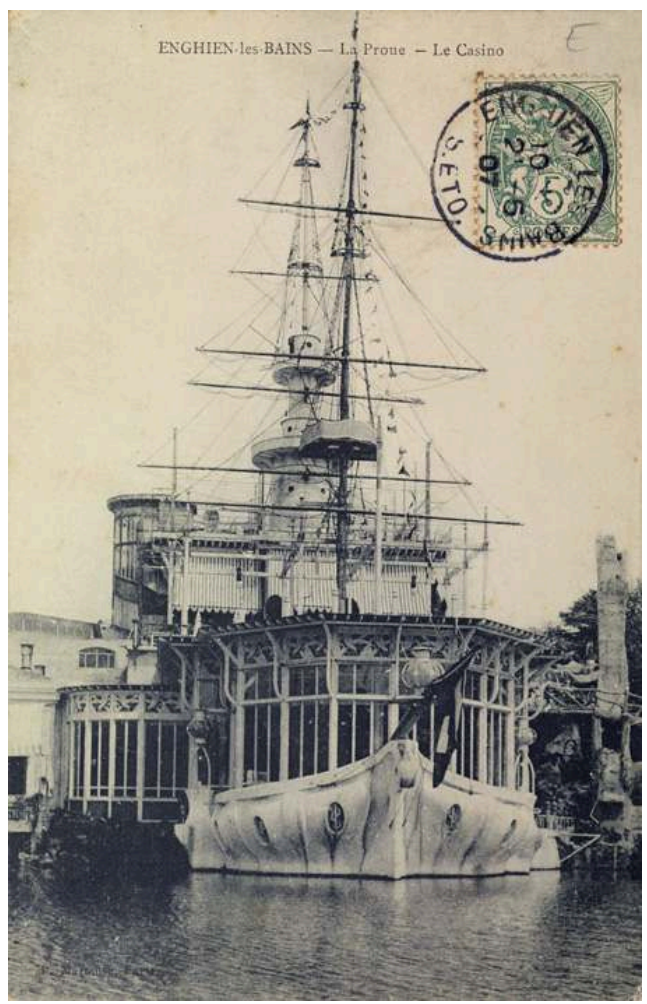

Le navire-casino vu du côté de « la proue », carte postale. AD 95

Repro. Vialles, Jean-Bernard. (c) Conseil régional d'Île-de-France, service Patrimoines et Inventaire, 2009. (base Mémoire : 20099500213) 


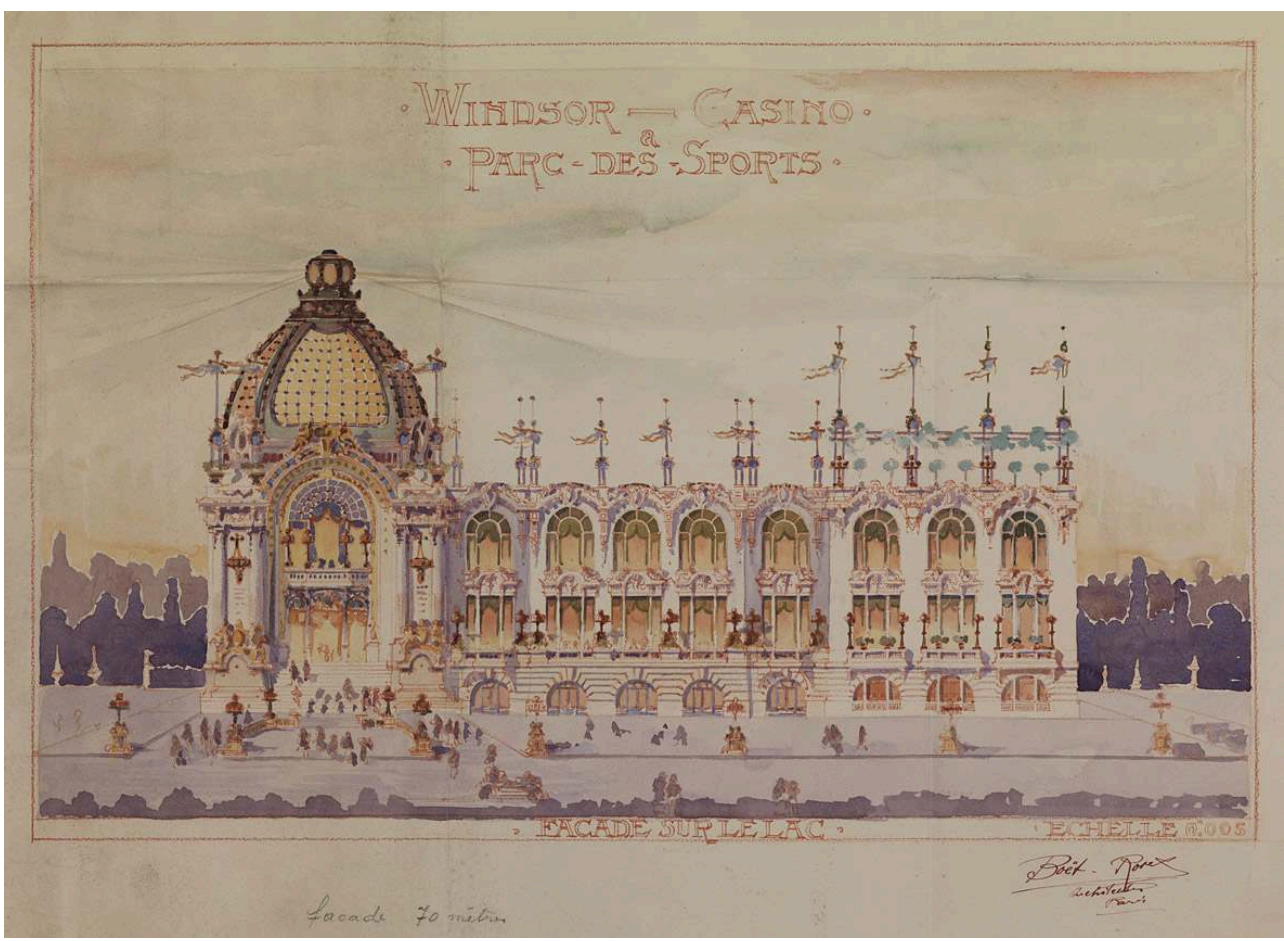

Projet pour le Windsor Casino par Boët et Roset, 1909, élévation sur le lac. Archives municipales d'Enghien-les-Bains.

Repro. Vialles, Jean-Bernard. (c) Conseil régional d'Île-de-France, service Patrimoines et Inventaire, 2009. (base Mémoire : 20099500134)

Dernière construction en lien direct avec le lac, une piscine est édifiée en 1957 par l'architecte Marcel Guilgot, ensemble élégant accompagné d'un jardin et d'un auditorium aujourd'hui disparu.

Enghien ville-lac? Au terme de cette approche essentiellement orientée vers l'influence d'un site, il semblerait que nous puissions conclure par l'affirmative. Toutefois il faut nuancer le propos. S'il est indubitable que le lac a été un élément primordial dans la création de la station thermale autant pour les premières implantations de bâtiments que pour l'élaboration d'un imaginaire lacustre, il faut souligner que dès 1850, date à laquelle la station devient commune, la ville va s'étendre bien au-delà avec de nouveaux quartiers. Mais ce dernier reste l'emblème de la ville. Dans l'édition du poème musical La Renaissance d'Enghien dédiée à Patenôtre-Desnoyer, député maire de 1919 à 1944, pour célébrer son activité édilitaire, c'est toujours une vue du lac qui est disposée en en-tête (fig. 14). 
Figure 14

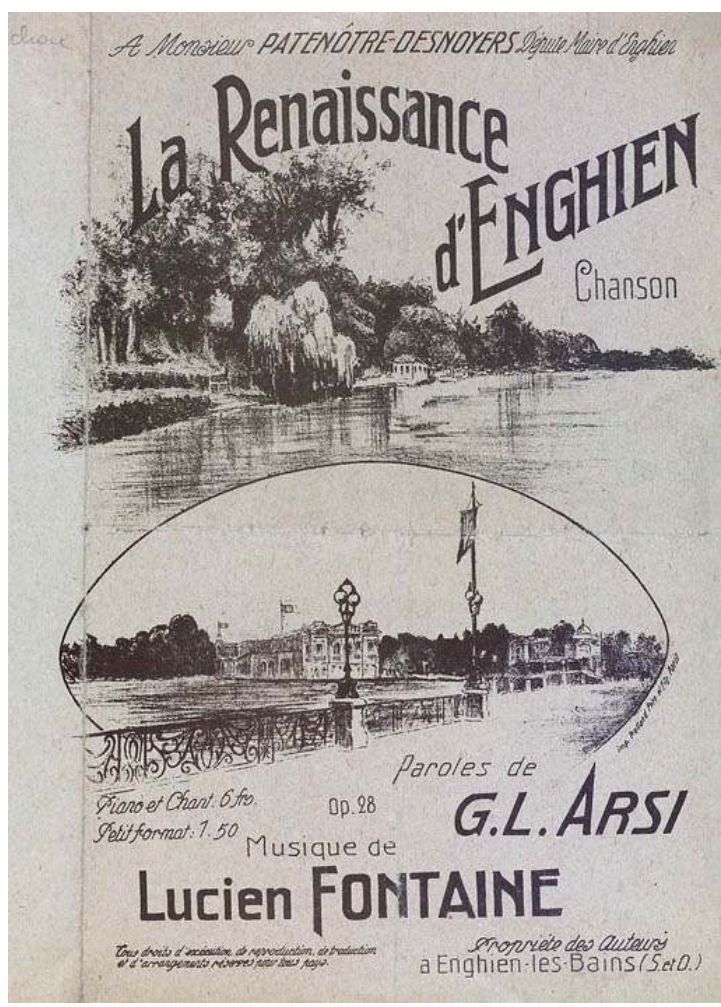

Couverture de la partition La Renaissance d'Enghien. Collection mairie d'Enghien.

Repro. Vialles, Jean-Bernard. (c) Conseil régional d'Île-de-France, service Patrimoines et Inventaire, 2009. (base Mémoire : 20099500576)

\section{NOTES}

1. - Cet article vient en complément de l'étude d'inventaire topographique sur l'aire d'étude d'Enghien-les-Bains, menée par le service Inventaire et Patrimoines au conseil régional d'Ile-deFrance et dont a été issu l'ouvrage: CUEILLE, Sophie. Enghien-les-Bains, architecture et décors. [Collection Images du Patrimoine, n²55]. Paris : Somogy Éditions d'Art, 2009.

2. - LONGCHAMP. Analyse des eaux minérales sulfureuses d'Enghien faite par ordre du gouvernement. Paris : librairie Cochard, 1826.

3. - ROUSSEAU, Jean-Jacques. Euvres complètes, sous la direction de B. Gagnebin et M. Raymond. Paris : Gallimard, bibliothèque de la Pléiade, 1959-1995, p. 152.

4. - Voir Julie et saint Preux sur le lac: http://www.herodote.net/_images/ julie_saintpreux_leman_crespy_maxi.jpg.

5. - En 1765, Jean-Jacques Rousseau résida six semaines en exil sur l'île (lac de Bienne, canton de Berne, au pied de la chaîne du Jura). Voir la Vue du lac de Bienne : http://www.culture.gouv.fr/ Wave/image/joconde/0373/m043703_0000679_p.jpg.

6. - CUEILLE, Sophie. Enghien-les-Bains, architecture et décors, op. cit., p. 5-6.

7. - LECOMTE, Jules. Le capitaine Sabord. Paris : Hippolyte Souverain éditeur, 1839. 
8. - Référence citée par GIRARDIN, Émile de et alii. Enghien et ses environs. Paris: Michel Levy frères, 1860, p. 32.

9. - Voir RENAULT, Delphine. Vichy, invitation à la promenade. Lyon : éditions Lieux dits, 2010.

10. - Voir La promenade sur le lac : http://www.lectura.fr/expositions/savoie/galeries/gal_chap1/ img06.jpg.

11. - RÉVILLÉ-PARISÉ, J.H. Une saison aux eaux minérales d'Enghien. Considérations hygiéniques et médicales sur cet établissement. Paris : Dentu, 1842, p. 163. Le médecin évoque une première visite sur les lieux et rapporte qu'il n'y avait alors que très peu d'habitations.

12. - LONGCHAMP, op cit.

13. - Avant de former une commune, une partie du territoire d'Enghien et donc du lac se trouvait sur la commune de Saint-Gratien, d'où le fait que parfois on trouve mention du « lac de SaintGratien ».

14. - PONSIN. Histoire des Bains. Enghien : 1910, p. 25. C'est depuis cet achat que le lac est entré dans la propriété des différentes sociétés thermales qui vont se succéder.

15. - AD 95, 2059/1 document imprimé et plan dans la liasse 38309.

16. - BnF, Cartes et Plans, GE C-3018 ou GE C-3747. Plans non datés.

17. - L'hôtel des Quatre Pavillons a été élevé en1822 par l'architecte Moreau à la demande des colonels de l'Empire Braque, Trobrian, Louvières et Bro, ce dernier ayant fait élever sa maison non loin de l'hôtel. Les deux bâtiments n'existent plus.

18. - Les aménagements successifs de cette île frapperont les imaginations. RévilléParisé évoque un jardin anglais et un " pavillon rustique imitant la forme d'un temple de l'Antiquité » où l'on peut dîner. Émile de Girardin en 1860 mentionne un « restaurant amphibie avec douze salons particuliers, un grand salon et une galerie ».

19. - CUEILLE, Sophie. Le Vésinet modèle français d'urbanisme paysager, 1858-1930. [Collection Cahiers du Patrimoine, $\mathrm{n}^{\circ} 17$ ]. Paris : Imprimerie nationale Éditions, 1989, rééd. 2002; Maisons-Laffitte, parc, paysage et villégiature, 1630-1930. [Cahiers du Patrimoine, n53]. Lyon : éditions Lieux Dits, 1999 ; réimpression 2005.

20. - Le lotissement sera réalisé plus simplement sur l'ensemble du pourtour du lac, y compris sur la chaussée où seront édifiées des maisons.

21. - Un plan (Plan du quartier d'Orléans et ses maisons avec jardin à l'anglaise) est conservé au musée Carnavalet Top GG 40G. Le lotissement a été étudié par R. Olson dans le cadre du CEAA architecture urbaine, École d'architecture de Paris Villemin, 1986. Voir le plan d'élévation $\mathrm{du}$ quartier d'Orléans au Petit Montrouge, 1830: http:// www.parismetropolitaine.fr/naissancedes20arrondissementsparisiens/img/gd/

2-2-50.jpg.

22. - Voir le plan de Marylebone Park: http://darkwing.uoregon.edu/ helphand/ parksoneimages/image64.jpg.

23. - Cet aspect urbain a été notamment abordé par Mark Girouard dans Des villes et des hommes, architecture et Société. Paris : Flammarion, 1987 (1985 pour l'édition originale anglaise). Les lotissements évoqués sont reproduits pages 265,276 et 278. Voir le Plan de Victoria Park : http:// manchesterhistory.net/longsight/VICTORIA/foundation.html

24. - AD 95, 2059/1. Il est précisé que les contrats entre les propriétaires et la société Péligot ont été passés devant Daloz et Thipaines Desanneaux, notaires à Paris.

25. - AD 95 2059/2.

26. - LEFEUVE, Charles. Le Tour de la Vallée : histoire et description de Montmorency. Paris : Dumoulin libraire-éditeur, 1856, p. 114. 
27. - On peut citer parmi les œuvres musicales répertoriées dans Gallica, BnF, La suite de valses «Le Lac d'Enghien », œuvre pour piano de J.H. Collet, 1891 ou «La Polka du lac », par Paul Courtois en 1878.

28. - Guide Joanne, 1856.

29. - RÉVILLÉ-PARISÉ, op. cit.

30. - GUINOT. Enghien et la vallée de Montmorency. Paris : E. Bourdin, 1847, p. 35.

31. - Voir Les promenades parisiennes, 1869: http://gallica.bnf.fr/ark:/12148/btv1b69384617/ f1.highres.

32. - Poésies de Léon Duvauchel 1869-1902. Paris : Alphonse Lemerre éditeur, 1905, p. 187-191.

33. - GIRARDIN, Émile de et alii, op. cit., p. 17.

34. - Guide Enghien et ses thermes. Vichy : imprimerie A. Wallon, 1868, p. 7 et 56.

35. - RÉVILLÉ-PARISÉ, op.cit, p. 158.

36. - Rappelons que Jean-Jacques Rousseau avait déjà présenté Robinson Crusoé comme «le plus heureux traité d'éducation naturelle » voire le seul ouvrage à lire... dans l'Émile ou de l'Éducation, 1762.

37. - Voir : http://gallica.bnf.fr/ark:/12148/bpt6k54912933/f1.highres.

38. - Le sujet a été abordé par CORBIN, Alain. Le Territoire du vide. L'Occident et le désir du rivage 1750-1840. Paris : Aubier, 1988.

39. - Bernardin de Saint-Pierre était un ami et suiveur de Jean-Jacques Rousseau et rappelons qu'il s'était fait construire une maison en Essonne sur une île au milieu de la Seine à hauteur de Corbeil. Voir aussi : http://www.culture.gouv.fr/Wave/image/joconde/0057/ m500202_03nu11247_p.jpg.

40. - RÉVILLÉ-PARISÉ, op. cit., p. 18-19.

41. - RÉVILLÉ-PARISÉ, op. cit., p. 160.

42. - Guide Enghien et ses thermes, op.cit., p. 51.

43. - VILLENEUVE, A. La sœur de Moïse. Chappe, 1859.

44. - BANVILLE, Théodore de. Les Parisiennes. Paris : Michel Lévy Frères, 1866.

45. - Voir la Ville de Shaffhouse, gravure aquarellée, vers 1825 : http://www.galerie-de-loes.ch/ images/oeuvres/9569212a.jpg.

46. - Vue de Kiew, gravure aquarellée, 1850 : http://www.antique-prints.de/shop/Media/Shop/ 8343.jpg.

47. - REVOIL, Benedict-Henri. Les Chasses dans l'Amérique du Nord. Alfred Mame \& Fils, 1883.

48. - CAPUS, Guillaume. À travers le voyage de Tamerlan. Asie centrale : voyage de la Sibérie occidentale. 1892. Voir la Vue de Petropavlovsk: http://russianhistoryblog.org/wp-content/uploads/2012/02/ Petropavlovsk-Kamchatka-1024x560.jpg.

49. - DELORD, T., FREMY, A., TEXIER, E. Les Petits Paris. Taxide, 1878.

50. - Guide Enghien et ses thermes, op. cit., p. 52.

51. - GONZALVÈS, Emmanuel. Le Vengeur du mari. Paris : Victor Benoît et $C^{\text {ie }}$ éditions illustrées, 1862, p. 69.

52. - BREHAT, Alfred de. Aventures d'un petit Parisien. Paris : J. Hetzel éditeur, 1862. La même image est reprise en 1892 dans Les Voyageurs involontaires, récit de Julien Biart où des jeunes gens découvrent la mer mais considèrent qu'ils connaissent déjà la mer puisqu'ils ont vu le lac d'Enghien. 


\section{RÉSUMÉS}

À la fois lac d'Elvire, lac Majeur, lac de Genève, terre chérie de l'auteur de l'Émile, l'un des plus jolis pays du monde, véritable miniature des grands lacs suisses, petite mer... Quel est ce lieu chanté par les plus belles plumes? Alfred de Musset, Théodore de Banville, Hector Malot, Alphonse Daudet, Guy de Maupassant, Alexandre Dumas, les frères Goncourt ont connu et évoqué le site, de même que nombre de romanciers oubliés. Thème décliné en poésie, en musique, référence géographique associée à Pékin, au Canada, en passant par la Sibérie occidentale... Enghien-les-Bains doit sans doute cette notoriété à sa proximité avec la capitale. Station thermale, mais également lieu de villégiature au bord d'un lac, elle a suscité bien des exercices littéraires où la surenchère de références, la métaphore et l'emphase promeuvent le site. Expérience urbaine d'avant-garde, colonie au dessin empruntant aux modèles anglais, tentative de réponse architecturale à la présence du lac, Enghien mérite une attention particulière.

At the same time Lake of Elvira, Lake Maggiore, Geneva Lake, beloved ground of the author of Émile, one of the most attractive countries of the world, the real miniature of the big Swiss lakes, the small sea... Which is this place sung under the most beautiful feathers ? Alfred de Musset, Théodore de Banville, Hector Malot, Alphonse Daudet, Guy de Maupassant, Alexandre Dumas, the brother Goncourt knew and evoqued the site, as well as number of forgotten novelist. Theme declined in poetry, in music, geographical reference associated in Pekin, in Canada by way of western Siberia... Enghien-les-Bains doubtless owes this fame to its nearness with the capital. Health resort, but also holiday resort at the edge of a lake it aroused many literary exercises where the repository higher bid, the metaphor and the bombast promote the site. Cutting edge urban experiment, colony to the drawing borrowing from the English models, architectural attempts at a reply in the presence of the lake, Enghien deserves a particular attention.

\section{INDEX}

Mots-clés : lac, villégiature, thermalisme, Enghien, Enghien-les-Bains

\section{AUTEUR}

\section{SOPHIE CUEILLE}

conservateur en chef du patrimoine, conservateur régional de l'Inventaire, service des Patrimoines, direction de la Culture et du Patrimoine, Collectivité territoriale de Corse sophie.cueille@ct-corse.fr 